activation involved in auditory speech perception in early schoolaged children using magnetoencephalography (MEG) that enables characterization of both the timing and location of activated cortical areas. We further explored the linkage between neural activation and behavioral linguistic skills. In line with previous EEG studies, the children showed clearly later and longer-lasting auditory responses than the adults. The most prominent activation was evoked between 200 and $400 \mathrm{~ms}$ in children, compared to the transient peak of activation at $\sim 100 \mathrm{~ms}$ in adults. Yet, judging by location and direction of current flow the cortical activation was similar in adults and children. Thus, at first glance, the neural activation in children resembled a stretched-out version of the adult response. However, in some of the children, also an adult-type response at $\sim 100 \mathrm{~ms}$ could be detected, although more often in the right hemisphere. When using simple speech and nonspeech sounds, sensitivity to sound-type emerged bilaterally at about $250 \mathrm{~ms}$, in contrast to the left-lateralized effect at $100 \mathrm{~ms}$ in adults. Interestingly, a shorter duration of the lefthemisphere $250-\mathrm{ms}$ response to auditory stimuli was linked with better reading skills. Our findings support the idea that the neural activations at $\sim 100 \mathrm{~ms}$ and $\sim 250 \mathrm{~ms}$ reflect distinct processes, the balance between which changes with development, with possible effect of hemisphere. Moreover, the prolonged activation at around $250 \mathrm{~ms}$ in the immature auditory cortex seems to be relevant for the cognitive development of the child.

doi:10.1016/j.ijpsycho.2012.06.180

\section{Auditory temporal processing in dyslexia: An ASSR study in young children}

S. Vanvooren ${ }^{\mathrm{a}}$, H. Poelmans ${ }^{\mathrm{a}}$, M. Hofmann ${ }^{\mathrm{a}}$, H. Luts ${ }^{\mathrm{a}}$, S. Dandache ${ }^{\mathrm{b}}$, P. Ghesquière ${ }^{b}, J_{\text {. Wouters }}{ }^{\mathrm{a}}$

${ }^{a}$ ExpORL, Dept. Neurosciences, KU Leuven, Belgium

${ }^{b}$ Research Unit Parenting and Special Education, KU Leuven, Belgium

Slow temporal modulations below $40 \mathrm{~Hz}$ have been shown to be important for speech understanding. Modulation frequencies near 4 and $20 \mathrm{~Hz}$ represent the rate by which syllables $( \pm 250 \mathrm{~ms})$ and phonemes ( $\pm 50 \mathrm{~ms})$ appear in speech, respectively.

It has been hypothesized that a neural deviance in the processing of these lower temporal modulations relates to the reading and spelling problems in dyslexia, a specific learning disorder. This deviance may cause subtle speech perception problems and could eventually lead to impaired literacy skills.

The neural correlates of temporal auditory processing can be investigated by recording Auditory Steady-State Responses (ASSRs) in the electroencephalogram (EEG). ASSRs measure the ability of the auditory system to follow the rate of temporal information and may therefore provide an objective measure to determine the sensitivity for important acoustical-phonological elements in language.

In the present longitudinal study, temporal auditory processing was investigated in a group of 45 children with and 45 children without an increased hereditary risk of dyslexia by means of ASSRs in combination with cognitive and psychophysical measures. All participating children attended their last year of nursery school at the moment of testing.

Multichannel ASSRs were recorded in a 64 electrode configuration using 100\% amplitude-modulated speech-weighted noise stimuli at 4, 20 and $80 \mathrm{~Hz}$. The $80 \mathrm{~Hz}$ ASSR is known to be evoked in the brainstem, while the $4 \mathrm{~Hz}$ and $20 \mathrm{~Hz}$ ASSRs are generated in the cortex.

Response strength is examined with response amplitudes and signal-to-noise ratios. In addition, hemispheric response asymmetry is assessed with a laterality index. The underlying neural sources are fitted using brain source analysis methods.
Preliminary results indicate differences between syllable and phoneme level for children with and children without an increased risk of dyslexia. Previous research demonstrated deviant processing on phoneme level in adults with dyslexia. Analyses are ongoing. Further results and implications will be discussed at the conference.

doi:10.1016/j.ijpsycho.2012.06.181

\section{Audiovisual integration of print and speech emerges when children learn to read: A developmental neuroimaging study}

\author{
S. Brem ${ }^{\mathrm{a}, \mathrm{b}}$, U. Richardson ${ }^{\mathrm{a}}$, S. Bach ${ }^{\mathrm{a}, \mathrm{b}}$, C. Hofstetter ${ }^{\mathrm{b}}$, \\ E. Martin ${ }^{\mathrm{c}, \mathrm{d}}$, D. Brandeis ${ }^{\mathrm{b}, \mathrm{d}, \mathrm{e}}$ \\ ${ }^{a}$ Agora Center, University of Jyväskylä, Finland \\ ${ }^{b}$ Department of Child and Adolescent Psychiatry, University of Zurich, \\ Switzerland \\ ${ }^{c}$ MR-Center, University Children's Hospital Zurich, Switzerland \\ ${ }^{d}$ Center for Integrative Human Physiology, University of Zurich, Zurich, \\ Switzerland \\ ${ }^{e}$ Department of Child and Adolescent Psychiatry and Psychotherapy, \\ Central Institute of Mental Health, Medical Faculty Mannheim/ \\ Heidelberg University, Mannheim, Germany
}

When children learn to read, the brain starts to integrate spoken and written language information. Integration and conceptual matching of incoming audiovisual information are highly automatized in good readers but deficient in poor readers (Blau et al. 2009), involve the superior temporal sulcus (STS) (van Atteveldt et al. 2004; Hocking and Price 2008) and start after around $380 \mathrm{~ms}$ (Raij et al. 2000). In this study we aimed to track the emergence and development of audiovisual integration from kindergarten to adulthood in normal and poor readers with functional magnetic resonance imaging (fMRI) and event-related potentials (ERPs).

Kindergarten children, normal and poor reading 2 nd graders as well as normal reading adults were examined when processing congruous and incongruous audiovisual speech and print information with fMRI ( $3 \mathrm{~T}$ ) and ERPs (64 channels). Incongruence effects determined by the statistical difference between incongruous and congruous audiovisual presentations are reported for each age group and for the differences between groups. For ERPs, amplitudes and topographies from 100 to $700 \mathrm{~ms}$ were analyzed. Random effect analyses were used to detect corresponding differences in the activation patterns of the fMRI data.

Audiovisual incongruence effects started to emerge in children after learning grapheme-phoneme correspondences and were pronounced in normal reading 2 nd graders and adults but were absent in poor reading 2 nd graders. The incongruence effect was found as a more pronounced activity to incongruous information in frontal and temporal regions in fMRI data and as a pronounced centro-parietal negativity in ERP data starting at around $380 \mathrm{~ms}$ in adults and slightly later in normal reading 2 nd graders.

In summary our results show that incongruous presentation of speech and print evokes an incongruency effect in fMRI and ERPs as soon as children's basic reading skills allow matching written to spoken information. The modulation of the incongruence effect by children's reading skills points to deficient integration of audiovisual information in poor readers.

Project supported by the European Commission's FP6, Marie Curie Excellence Grants (MEXT-CE-2004-014203) and the Hartmann MüllerStiftung for medical research (project no. 1252) Zürich.

doi:10.1016/j.ijpsycho.2012.06.183 